\title{
Study Population Subgroup
}

National Cancer Institute

\section{Source}

National Cancer Institute. Study Population Subgroup. NCI Thesaurus. Code C71468.

Relatively uniform groups (e.g. etiologically and pathogenetically more homogeneous, racial, ethnic, or gender-specific, or the disease- or condition-specific) of study subjects formed within a study population for a separate analysis. The selection of the subgroups, methods of subgroup analysis, and anticipated difference in observation outcome(s) have to be defined and justified in the study protocol and based on known biological mechanisms, and/or findings in previous studies. 